Bangladesh J. Pl. Breed. Genet., 26(2): 23-32, 2013

\title{
COMBINING ABILITY EFFECTS AND PER-SE PERFORMANCES OF MORPHO REPRODUCTIVE TRAITS IN RICE (Oryza sativa L.)
}

\author{
M. A. Islam ${ }^{1}$, M. A. K. Mian, M. G. Rasul, Q. A. Khaliq ${ }^{2}$ and \\ M. K. Bashar ${ }^{3}$ \\ Department of Genetics and Plant Breeding \\ Bangabandhu Sheikh Mujibur Rahman Agricultural University \\ Gazipur 1706, Bangladesh
}

\begin{abstract}
To study combining ability effects of developed CMS and restorer lines an experiment was carried out at the experimental farm, Department of Genetics and Plant Breeding, Bangabandhu Sheikh Mujibur Rahman Agricultural University, Salna, Gazipur during Aman 2011 and Boro 2011-12 following RCBD design through line $\times$ tester analysis in three replications. Five known CMS lines and sixteen developed Restorer lines were considered for through line $\times$ tester analysis. Among sixteen restorer lines, only four showed significant negative GCA effects for all these three traits for earliness. The estimated of gca effects of parents indicated that seven parents and sixteen crosses contributed highly significant negative effects for plant height which were responsible for dwarfing character. Fifteen crosses and eight parents showed significant negative sca estimates for days to first flowering and twenty crosses for maturity. Considering both SCA effects and per se performances for days to $1^{\text {st }}$ flowering and maturity; the crosses of IR 58025A with RG-BU08005R, RG-BU08-006R, RG-BU08-016R, RG-BU08-018R and RG-BU08-025R were found as the best specific combiner. Among 80 crosses fifty two crosses showed significant positive SCA effects along with above average perse performances for grain yield. Ten crosses of IR 58025A, seven crosses of GAN46A, fourteen crosses of IR 62829A, nine crosses of IR 68888A and ten crosses of BRRI 1A showed significant positive SCA effects along with mean values resulting significant positive SCA effects and above average perse performances with all five CMS lines for grain yield.
\end{abstract}

Keywords: Rice (Oryza sativa L.); GCA effects; SCA effects; per se performances

\section{INTRODUCTION}

Combining ability is a powerful tool in identifying the best combiners that may be used in crosses either to exploit heterosis or to accumulate fixable genes and obtain desirable segregates. Evaluation of inbred lines is the prerequisite for any hybrid program. Combining ability analysis is one of the powerful available evaluation tools to estimate the combining ability variance and effects for selecting the desirable parents and crosses for exploitation of heterosis. Combining ability variance is usually used for the estimation of genetic control of a specific trait (Islam, 2009). The estimates of additive and non-additive gene action through this technique may be useful in determining the possibility of commercial exploitation of heterosis and isolation of pure line. Hybrid rice offers an opportunity to boost the yield potential of rice. Hybrid varieties have a

\footnotetext{
${ }^{1}$ Dept of Genetics and Plant Breeding, EXIM Bank Agricultural University, Chapainawabgonj, Bangladesh; ${ }^{2}$ Department of Agronomy, Bangabandhu Sheikh Mujibur Rahman Agricultural University, Gazipur 1706, Bangladesh; ${ }^{3}$ Country Manager, International Potato Research Centre (CIP), Bangladesh
} 
yield advantage of 15-20\% over conventional high-yielding varieties (Virmani et al. 1993). Hybrid rice research now concentrates on the conversion and identification of stable local cytoplasmic male sterile (CMS) lines and effective restorers from local elite lines through repeated backcrossing. To exploit maximum heterosis using male sterility system in hybrid breeding program, the combining ability estimation of different male sterile and restorer lines and their crosses are needed to be estimated. The development and use of hybrid rice varieties on commercial scale utilizing CMS fertility restoration system has proved to be one of the mile stones in the history of rice improvement.

Combining ability will help to understand the genetic architecture of various characters that enable the breeder to design effective breeding plan for future up-gradation of the existing materials. This information may also be useful to breeders for genetic improvement of the existing genotypes on the basis of the performance in various hybrid combinations (Islam, 2009). Breeding strategies based on selection of hybrids require expected level of heterosis as well as the specific combining ability. In breeding high yielding varieties of crop plant, the breeders often face with the problem of selecting parents and crosses. Combining ability analysis is one of the powerful tools available to estimate the combining ability effects and aids in selecting the desirable parents and crosses for the exploitation of heterosis. Line $\mathrm{x}$ tester analysis provides information about general combining ability (GCA) effects of parents and is helpful in estimating various types of gene actions (Zhang et al. 2002). Therefore, the present investigation was carried out to estimate combining ability effects for yield components involving CMS and restorer lines. Considering the above idea the present investigation was undertaken with the objective to determine the extent of combining ability variances in some morpho-reproductive traits for selection of suitable parents.

\section{MATERIALS AND METHODS}

An experiment was carried to study combining ability effects of developed CMS and restorer lines at the experimental farm, Department of Genetics and Plant Breeding, Bangabandhu Sheikh Mujibur Rahman Agricultural University, Gazipur during Aman 2011 and Boro 2011-12 following RCBD design in three replications. Five known CMS lines and sixteen suspected Rlines were considered for line $\times$ tester analysis. Analysis of variances for general and specific combining ability (GCA and SCA) was estimated according to line $\times$ tester method (Kempthorne, 1957). Five female parents ( IR 58025A, BRRI 1A, GAN46A, IR 68888A and IR 62820A) and sixteen male parents ( RG-BU08-001R, RG-BU08-002R, RG-BU08-005R, RG-BU08-006R, RGBU08-007R, RG-BU08-013R, RG-BU08-016R, RG-BU08-018R, RG-BU08-025R, RG-BU08034R，RG-BU08-038R，RG-BU08-046R，RG-BU08-057R，RG-BU08-063R，RG-BU08-097R and RG-BU08-105R) were used in the experiment. Data were collected from 10 hills of each genotype on 10 randomly selected individual plant basis are Data were collected from 10 hills of each genotype on 10 randomly selected individual plant basis are plant height, days to $1^{\text {st }}$ flowering, days to maturity and grain yield (ton/ha). Data obtained for each character was subjected to the analysis of variance following three replicated randomized complete block design by using GENSTAT program.

\section{RESULTS AND DISCUSSION}

\section{General combining ability effects}

The general combining ability effects of the parents in the present study have brought to the light the parents with high gca effects for five different traits. The estimated of gca effects of parents indicated that the parent, RG-BU 08-001R, RG-BU 08-002R, RG-BU 08-0046R, RG-BU 08-0057R, RG-BU 08-0097R, BRRI 1A and IR62829A contributed highly significant negative effects (Table 1). These facts indicated that these parents possessed more negative alleles for the dwarf stature. Perse performance also supported that RG-BU 08-001R $(89.03 \mathrm{~cm})$, RG-BU 08006R (96.72 cm), RG-BU 08-057R (90.39 cm), RG-BU 08-097R $(81.39 \mathrm{~cm})$ and BRRI 1A $(90.59 \mathrm{~cm})$ except RG-BU 08-002R $(103.69 \mathrm{~cm})$ and IR62829A $(108.57 \mathrm{~cm})$ these parents were dwarf in stature. Although general GCA effects found significant negative but perse performances 
did not correlate with RG-BU08-002R $(103.69 \mathrm{~cm})$ and IR62829A $(108.57 \mathrm{~cm})$ which might be due to pseudo recessive gene effect or wide environmental fluctuation. Therefore, RG-BU 08001R, RG-BU 08-046R, RG-BU 08-057R, RG-BU 08-097R and BRRI 1A are potential parents and have highly significant GCA effect in the desirable direction (negative direction) for plant height. These findings are in accordance with $\mathrm{Su}$ and Chen (2006).

Out of sixteen restorer lines eight restorer lines showed significant negative effects for days to $1^{\text {st }}$ flowering, and five showed significant negative effects for days to maturity. But among these 16 restorer lines, only four showed significant nigative GCA effects for both first flowering and maturity. The restorer lines showing significant nigative gca effects for these traits are RG-BU 08-005R (-7.43**, -7.590**), RG-BU 08-006R (-6.073**, -7.412**), RG-BU 08-007R (-3.743*, $7.564 * *)$, RG-BU 08-097R $\left(-8.743^{* *},-5.532 * *\right)$. Such lines could be used as male parent for development of early maturing hybrids in rice. Rao et al. (2006) observed that IR58025A and IR62829A were good general combiners for earliness, grain yield per plant and per day productivity. Compared to BRRI dhan29 these parents (IR 62829A and GAN 46A) matured 27 \pm 2 days earlier. So, RG-BU 08-005R, RG-BU 08-006R, RG-BU 08-007R and RG-BU 08-057R might be used in the heterosis breeding. As general combining ability (GCA) effects found significant negative and their perse performances were comparatively lower; therefore, these parents might be used as suitable parents to develop short duration hybrid variety. These findings are in accordance with Won \& Yoshida 2000.

Significant positive gca effects was found in RG-BU 08-002R (0.879**), RG-BU 08005R (0.439*), RG-BU 08-006R (1.069**), RG-BU 08-007R (0.449*), RG-BU 08-013R $(0.449 *)$, RG-BU 08-016R (0.449*), RG-BU 08-034R (0.909**), IR 58025A (0.074**) and IR 68888A $\left(0.346^{* *}\right)$. Perse performances revealed that seven pollen parents RG-BU 08-002R (5.98 t/ha), RG-BU 08-005R (5.54 t/ha), RG-BU 08-006R (6.71 t/ha), RG-BU 08-007R (5.55 t/ha), RGBU 08-013R (5.55 t/ha), RG-BU 08-016R (5.55 t/ha), RG-BU 08-034R (6.01 t/ha) and 2 CMS lines IR 58025A (2.40 t/ha) and IR 68888A (2.66 t/ha) were superior to others. These facts indicated that among 21 parents these nine parents possessed more positive alleles for the increase of grain yield. Dorosti et al. 2006, Chen et al. 2002 and Faiz et al. 2006 observed good general combiner CMS lines for grain yield along with other yield contributing characters in rice. So, among the male parents, RG-BU 08-002R, RG-BU 08-005R, RG-BU 08-006R, RG-BU 08-007R, RG-BU 08-013R, RG-BU 08-016R and RG-BU 08-034R were the best general combiner for grain yield due to highly significant positive GCA effects. On the other hand RG-BU 08-025R ($\left.2.141^{* *}, 2.96 \mathrm{t} / \mathrm{ha}\right), \mathrm{RG}-\mathrm{BU}$ 08-038R (-0.431*, 4.67 t/ha), RG-BU 08-046R (-0.341*, 4.76 t/ha), RG-BU 08-057R (-1.231*, $3.87 \mathrm{t} / \mathrm{ha})$, and RG-BU 08-097R (-1.311*, $3.79 \mathrm{t} / \mathrm{ha})$ as well as CMS parents IR 62829A $(-0.154 * *, 2.16 \mathrm{t} / \mathrm{ha})$ and BRRI 1A $(-0.184 * *, 2.13 \mathrm{t} / \mathrm{ha})$ showed highly significant negative general combining ability effects. Banumathy and Thiyagarajan 2005 found similar results while studying GCA effects in rice

\section{A. Specific combining ability effects}

The specific combining ability effects of the trait plant height is considered as an important character to select a hybrid. Among the F1s a total of 16 crosses were found having significant negative heterosis for plant height. Where three crosses of IR 58025A, three crosses of BRRI 1A, four crosses of GAN46A, two crosses of IR 68888A and four crosses of IR 62820A exhinited significant negative heterosis for plant height. This result indicates that the following croosses are semi dwarf in nature. The crosses of IR58025A showed significant positive specific combining ability effects with RG-BU08-006R \& RG-BU08-034R (Table 2). As these crosses showed highly significant positive SCA effects and above average perse performances, might not be selected as suitable hybrid. The crosses of GAN46A also found significant positive SCA effects and above average perse performances with RG-BU08-007R, RG-BU08-018R \& RGBU08-105R. The crosses of IR68888A showed significant positive SCA effects and above average perse performances with RG-BU08-002R, RG-BU08-006R, RG-BU 08-018R and RGBU08-016R which can be considered as good specific combination for tallness. 
Table 1. GCA effects of parents for different yield and yield contributing character of rice

\begin{tabular}{|c|c|c|c|c|c|c|c|c|}
\hline $\begin{array}{ll}\text { Source } & \text { of } \\
\text { Variation } & \end{array}$ & \multicolumn{2}{|c|}{ Plant height (cm) } & \multicolumn{2}{|c|}{ Days first flowering } & \multicolumn{2}{|c|}{ Days to maturity } & \multicolumn{2}{|c|}{ Yield (ton/ha) } \\
\hline Lines & gi/gj & Mean & gi/gj & mean & gi/gj & Mean & gi/gj & Mean \\
\hline IR 58025A & -0.466 & $89.02 \pm 12.07$ & 0.824 & $106.66 \pm 1.12$ & -2.020 & $139.66 \pm 2.45$ & $0.074^{* *}$ & $2.30 \pm 0.04$ \\
\hline IR 62829A & -2.797 & $90.59 \pm 12.07$ & $-2.996^{*}$ & $102.84 \pm 1.12$ & -0.170 & $141.51 \pm 2.45$ & $-0.154 * *$ & $2.16 \pm 0.04$ \\
\hline GAN 46 A & 6.905 & $93.81 \pm 12.07$ & $-2.236^{*}$ & $103.60 \pm 1.12$ & $-5.740 * *$ & $135.94 \pm 2.45$ & 0.016 & $2.32 \pm 0.04$ \\
\hline IR 68888A & 0.194 & $107.73 \pm 12.07$ & -0.706 & $105.13 \pm 1.12$ & $2.660^{*}$ & $144.34 \pm 2.45$ & $0.346^{* *}$ & $2.66 \pm 0.04$ \\
\hline BRRI 1 A & -3.836 & $108.57 \pm 12.07$ & $5.114 * *$ & $110.95 \pm 1.12$ & $5.270 * *$ & $146.95 \pm 2.45$ & $-0.184 * *$ & $2.13 \pm 0.04$ \\
\hline SE (gi) & & 12.074 & & 1.129 & & 2.455 & & 0.023 \\
\hline $\mathrm{SE}(\mathrm{gi}-\mathrm{gj})$ & & 30.185 & & 2.222 & & 3.387 & & 0.057 \\
\hline \multicolumn{9}{|l|}{ Testers } \\
\hline $\begin{array}{l}\text { RG-BU 08-001 R } \\
\end{array}$ & $-5.123 * *$ & $89.03 \pm 2.27$ & -2.403 & $103.01 \pm 3.48$ & $5.436 * *$ & $146.34 \pm 5.04$ & 0.389 & $5.49 \pm 0.784$ \\
\hline RG-BU 08-002 R & $-5.823 * *$ & $103.69 \pm 2.27$ & $-4.743 *$ & $100.67 \pm 3.48$ & 1.106 & $142.01 \pm 5.04$ & $0.879 * *$ & $5.98 \pm 0.784$ \\
\hline RG-BU 08-005 R & -1.788 & $99.40 \pm 2.27$ & $-7.743 * *$ & $97.67 \pm 3.48$ & $-7.590 * *$ & $133.34 \pm 5.04$ & $0.439 *$ & $5.54 \pm 0.784$ \\
\hline RG-BU 08-006 R & $12.536 * *$ & $96.72 \pm 2.27$ & $-6.073 * *$ & $99.34 \pm 3.48$ & $-7.412 * *$ & $133.37 \pm 5.04$ & $1.609 *$ & $6.71 \pm 0.784$ \\
\hline RG-BU 08-007 R & -1.394 & $136.52 \pm 2.27$ & $-3.743 *$ & $101.67 \pm 3.48$ & $-7.564 * *$ & $133.02 \pm 5.04$ & $0.449 *$ & $5.55 \pm 0.784$ \\
\hline RG-BU 08-013 R & -1.063 & $98.54 \pm 2.27$ & $4.098^{*}$ & $109.51 \pm 3.48$ & -1.064 & $139.84 \pm 5.04$ & $0.449 *$ & $5.55 \pm 0.784$ \\
\hline RG-BU 08-016 R & -0.188 & $99.23 \pm 2.27$ & $11.928 * *$ & $117.34 \pm 3.48$ & $5.436 * *$ & $146.34 \pm 5.04$ & $0.449 *$ & $5.55 \pm 0.784$ \\
\hline RG-BU 08-018 R & 0.320 & $91.23 \pm 2.27$ & $4.598 *$ & $110.01 \pm 3.48$ & 0.106 & $141.01 \pm 5.04$ & 0.109 & $5.21 \pm 0.784$ \\
\hline RG-BU 08-025 R & 0.511 & $86.79 \pm 2.27$ & $-3.743 *$ & $101.67 \pm 3.48$ & -2.564 & $138.34 \pm 5.04$ & $-2.141 * *$ & $2.96 \pm 0.784$ \\
\hline RG-BU 08-034 R & $10.682 * *$ & $92.94 \pm 2.27$ & 11.258 ** & $116.67 \pm 3.48$ & -2.694 & $138.21 \pm 5.04$ & $0.909 * *$ & $6.01 \pm 0.784$ \\
\hline RG-BU 08-038 R & 0.199 & $96.11 \pm 2.27$ & $9.598 * *$ & $115.01 \pm 3.48$ & $5.436^{* *}$ & $146.34 \pm 5.04$ & $-0.431 *$ & $4.67 \pm 0.784$ \\
\hline RG-BU 08-046 R & $-3.282 *$ & $96.13 \pm 2.27$ & $-9.073 * *$ & $96.34 \pm 3.48$ & 7.436 ** & $148.34 \pm 5.04$ & -0.341 & $4.76 \pm 0.784$ \\
\hline RG-BU 08-057 R & $-7.027 * *$ & $100.39 \pm 2.27$ & -1.403 & $104.01 \pm 3.48$ & $-7.564 * *$ & $133.34 \pm 5.04$ & $-1.231 * *$ & $3.87 \pm 0.784$ \\
\hline RG-BU 08-063 R & $2.580^{*}$ & $87.41 \pm 2.27$ & $-6.073 * *$ & $99.34 \pm 3.48$ & -2.564 & $138.34 \pm 5.04$ & -0.101 & $5.00 \pm 0.784$ \\
\hline RG-BU 08-097 R & $-4.083 *$ & $81.39 \pm 2.27$ & $-8.743 * *$ & $96.67 \pm 3.48$ & $-5.532 * *$ & $135.31 \pm 5.04$ & $-1.311 * *$ & $3.79 \pm 0.784$ \\
\hline RG-BU 08-105 R & $2.942 *$ & $97.95 \pm 2.27$ & $12.258 * *$ & $117.67 \pm 3.48$ & $10.106^{* * *}$ & $151.01 \pm 5.04$ & -0.121 & $4.98 \pm 0.784$ \\
\hline 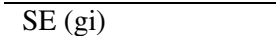 & & 2.278 & & 3.483 & & 3.581 & & 0.426 \\
\hline $\mathrm{SE}(\mathrm{gi}-\mathrm{gj})$ & & 4.594 & & 5.730 & & 5.039 & & 0.784 \\
\hline
\end{tabular}


Table 2. SCA effects (Sij) vis-à-vis per-se mean performance of hybrids for plant height $(\mathrm{cm})$ in $80 \mathrm{~F}_{1}$ hybrids

\begin{tabular}{|c|c|c|c|c|c|c|c|c|c|c|}
\hline \multirow{3}{*}{$\begin{array}{l}\text { Line } \\
\qquad \text { Testers }\end{array}$} & \multicolumn{10}{|c|}{ Plant height } \\
\hline & \multicolumn{2}{|c|}{ IR 58025A } & \multicolumn{2}{|c|}{ GAN 46A } & \multicolumn{2}{|c|}{ IR 62829A } & \multicolumn{2}{|c|}{ IR 68888A } & \multicolumn{2}{|c|}{ BRRI 1A } \\
\hline & Sij effect & mean & Sij effect & mean & Sij effect & mean & Sij effect & mean & Sij effect & Mean \\
\hline RG-BU 08-001 R & -4.98 & $98.79 \pm 7.26$ & -4.28 & $99.49 \pm 7.78$ & $-9.95 * *$ & $93.82 \pm 7.85$ & $-5.93 *$ & $92.84 \pm 7.39$ & -3.45 & $107.32 \pm 7.25$ \\
\hline RG-BU 08-002 R & $-5.50 *$ & $98.27 \pm 7.26$ & -3.91 & $99.86 \pm 7.78$ & $-8.86 * *$ & $94.91 \pm 7.85$ & 4.44 & $108.22 \pm 7.39$ & $-15.27 * *$ & $88.50 \pm 7.25$ \\
\hline RG-BU 08-005 R & -2.74 & $101.03 \pm 7.26$ & $-7.50 *$ & $96.27 \pm 7.78$ & $6.60 *$ & $110.38 \pm 7.85$ & -1.01 & $102.76 \pm 7.39$ & -4.28 & $99.49 \pm 7.25$ \\
\hline RG-BU 08-006 R & $10.71 * *$ & $114.49 \pm 7.26$ & 1.59 & $105.37 \pm 7.78$ & $46.40 * *$ & $150.18 \pm 7.85$ & $7.88 * *$ & $111.66 \pm 7.39$ & -3.91 & $99.86 \pm 7.25$ \\
\hline RG-BU 08-007 R & -0.34 & $103.43 \pm 7.26$ & 4.87 & $108.65 \pm 7.78$ & -2.31 & $101.46 \pm 7.85$ & -1.67 & $102.10 \pm 7.39$ & $-7.50 *$ & $96.27 \pm 7.25$ \\
\hline RG-BU 08-013 R & -3.49 & $100.28 \pm 7.26$ & $-15.64 * *$ & $88.13 \pm 7.78$ & $12.34 * *$ & $116.12 \pm 7.85$ & -0.11 & $103.66 \pm 7.39$ & 1.59 & $105.37 \pm 7.25$ \\
\hline RG-BU 08-016 R & -0.74 & $103.03 \pm 7.26$ & -3.54 & $100.23 \pm 7.78$ & -3.47 & $100.30 \pm 7.85$ & 1.95 & $105.73 \pm 7.39$ & 4.87 & $108.65 \pm 7.25$ \\
\hline RG-BU 08-018 R & 1.06 & $104.84 \pm 7.26$ & 4.76 & $108.54 \pm 7.78$ & $8.05 * *$ & $111.83 \pm 7.85$ & 3.36 & $107.14 \pm 7.39$ & $-15.64 * *$ & $88.13 \pm 7.25$ \\
\hline RG-BU 08-025 R & 0.10 & $103.88 \pm 7.26$ & -1.91 & $101.86 \pm 7.78$ & $5.37 *$ & $109.15 \pm 7.85$ & 2.53 & $106.31 \pm 7.39$ & -3.54 & $100.23 \pm 7.25$ \\
\hline RG-BU 08-034 R & $8.60 * *$ & $112.38 \pm 7.26$ & -1.75 & $102.02 \pm 7.78$ & $45.17 * *$ & $148.95 \pm 7.85$ & -3.38 & $100.39 \pm 7.39$ & 4.76 & $108.54 \pm 7.25$ \\
\hline RG-BU 08-038 R & -0.20 & $103.57 \pm 7.26$ & -2.21 & $101.56 \pm 7.78$ & $9.04 *$ & $112.82 \pm 7.85$ & -3.71 & $100.06 \pm 7.39$ & -1.91 & $101.86 \pm 7.25$ \\
\hline RG-BU 08-046 R & -3.93 & $99.84 \pm 7.26$ & -7.22 & $96.55 \pm 7.78$ & $-5.74 *$ & $98.03 \pm 7.85$ & 2.25 & $106.03 \pm 7.39$ & -1.75 & $102.02 \pm 7.25$ \\
\hline RG-BU 08-057 R & $-6.56 *$ & $97.21 \pm 7.26$ & -4.25 & $99.52 \pm 7.78$ & $-17.54 * *$ & $86.23 \pm 7.85$ & -4.55 & $99.22 \pm 7.39$ & -2.21 & $101.56 \pm 7.25$ \\
\hline RG-BU 08-063 R & 1.71 & $105.49 \pm 7.26$ & -2.62 & $101.15 \pm 7.78$ & $17.52 * *$ & $121.30 \pm 7.85$ & 3.51 & $107.29 \pm 7.39$ & $-7.22 *$ & $96.55 \pm 7.25$ \\
\hline RG-BU 08-097 R & $-5.38 *$ & $99.39 \pm 7.26$ & $-5.88 *$ & $97.89 \pm 7.78$ & $-5.66 * *$ & $98.44 \pm 7.85$ & $-6.55^{*}$ & $97.22 \pm 7.39$ & -4.25 & $99.52 \pm 7.25$ \\
\hline RG-BU 08-105 R & 3.24 & $107.02 \pm 7.26$ & 4.78 & $108.56 \pm 7.78$ & $7.19 *$ & $110.97 \pm 7.85$ & 2.10 & $105.88 \pm 7.39$ & -2.62 & $101.15 \pm 7.25$ \\
\hline Mean & & 103.31 & & 100.98 & & 110.68 & & 103.97 & & 99.94 \\
\hline $\boldsymbol{S E}(s i j)$ & 3.113 & & & & & & & & & \\
\hline SEd (Sij-Sik) & 5.153 & & & & & & & & & \\
\hline SEd (Sij-Skj) & 7.561 & & & & & & & & & \\
\hline $\mathrm{t}=\operatorname{Sij} / S E I(S i j)$ & $r d f=$ & & & & & & & & & \\
\hline
\end{tabular}

${ }^{*} \mathrm{p}=0.05,{ }^{*} \mathrm{p}=0.01$ and $^{\mathrm{ns}}=$ non-significant 
Table 3. SCA effects (Sij) vis-à-vis per-se mean performance of hybrids for days to first flowering in $80 \mathrm{~F}_{1}$ hybrids

\begin{tabular}{|c|c|c|c|c|c|c|c|c|c|c|}
\hline \multirow{3}{*}{$\begin{array}{l}\text { Line } \\
\text { Testers }\end{array}$} & \multicolumn{10}{|c|}{ Daysto first flowering } \\
\hline & \multicolumn{2}{|c|}{ IR 58025A } & \multicolumn{2}{|c|}{ GAN 46A } & \multicolumn{2}{|c|}{ IR 62829A } & \multicolumn{2}{|c|}{ IR 68888A } & \multicolumn{2}{|c|}{ BRRI 1A } \\
\hline & Sij effect & mean & Sij effect & mean & Sij effect & mean & Sij effect & mean & Sij effect & Mean \\
\hline RG-BU 08-001 R & $-4.214 *$ & $105.90 \pm 2.63$ & $4.450^{*}$ & $114.56 \pm 3.63$ & 0.12 & $114.56 \pm 3.63$ & $-5.54 *$ & $110.23 \pm 3.63$ & $4.450^{*}$ & $104.56 \pm 3.63$ \\
\hline RG-BU 08-002 R & -1.550 & $108.56 \pm 2.63$ & 0.340 & $110.45 \pm 3.63$ & -0.88 & $110.45 \pm 3.63$ & $7.12 *$ & $109.23 \pm 3.63$ & 0.340 & $117.23 \pm 3.63$ \\
\hline RG-BU 08-005 R & $-6.547 *$ & $103.56 \pm 2.63$ & -0.880 & $109.23 \pm 3.63$ & -1.88 & $109.23 \pm 3.63$ & $9.12 *$ & $108.23 \pm 3.63$ & -0.880 & $119.23 \pm 3.63$ \\
\hline RG-BU 08-006 R & $-8.650 *$ & $101.46 \pm 3.63$ & 0.570 & $110.68 \pm 3.63$ & $-4.88 *$ & $102.23 \pm 3.63$ & $-13.21 * *$ & $107.23 \pm 3.63$ & $-17.880^{* *}$ & $96.90 \pm 3.63$ \\
\hline RG-BU 08-007 R & 2.340 & $112.45 \pm 3.63$ & $-5.880^{*}$ & $104.23 \pm 3.63$ & -1.88 & $111.23 \pm 3.63$ & 3.12 & $108.23 \pm 3.63$ & 1.120 & $113.23 \pm 3.63$ \\
\hline RG-BU 08-013 R & $-7.547 *$ & $102.56 \pm 2.63$ & -3.214 & $106.90 \pm 3.63$ & 3.12 & $107.90 \pm 3.63$ & 3.12 & $113.23 \pm 3.63$ & -2.214 & $113.23 \pm 3.63$ \\
\hline RG-BU 08-016 R & $-9.547 *$ & $100.56 \pm 2.63$ & $-9.547 *$ & $100.56 \pm 3.63$ & 2.12 & $110.23 \pm 3.63$ & $6.13 *$ & $112.23 \pm 3.63$ & 0.120 & $116.23 \pm 3.63$ \\
\hline RG-BU 08-018 R & $-7.880 *$ & $102.23 \pm 2.63$ & $-7.880 *$ & $102.23 \pm 3.63$ & -1.88 & $113.56 \pm 3.63$ & $8.78^{*}$ & $108.23 \pm 3.63$ & 3.453 & $118.23 \pm 3.63$ \\
\hline RG-BU 08-025 R & $-5.547 *$ & $104.56 \pm 2.63$ & $-5.547 *$ & $104.56 \pm 3.63$ & 0.12 & $120.23 \pm 3.63$ & 2.78 & $110.23 \pm 3.63$ & $10.120 * *$ & $112.90 \pm 3.63$ \\
\hline RG-BU 08-034 R & -3.214 & $106.90 \pm 2.63$ & -3.214 & $106.90 \pm 3.63$ & 1.12 & $113.23 \pm 3.63$ & -1.21 & $111.23 \pm 3.63$ & 3.120 & $108.90 \pm 3.63$ \\
\hline RG-BU 08-038 R & -0.660 & $109.45 \pm 2.63$ & -0.660 & $109.45 \pm 3.63$ & 2.12 & $113.23 \pm 3.63$ & -2.54 & $112.23 \pm 3.63$ & 3.120 & $107.56 \pm 3.63$ \\
\hline RG-BU 08-046 R & -0.214 & $109.90 \pm 2.63$ & -0.214 & $109.90 \pm 3.63$ & 0.12 & $116.23 \pm 3.63$ & 0.12 & $110.23 \pm 3.63$ & $6.120^{*}$ & $110.23 \pm 3.63$ \\
\hline RG-BU 08-057 R & 3.120 & $113.23 \pm 2.63$ & 3.120 & $113.23 \pm 3.63$ & -0.88 & $118.23 \pm 3.63$ & -0.88 & $109.23 \pm 3.63$ & $8.120^{*}$ & $109.23 \pm 3.63$ \\
\hline RG-BU 08-063 R & 2.453 & $112.56 \pm 2.63$ & 2.453 & $112.56 \pm 3.63$ & $5.12 *$ & $112.90 \pm 3.63$ & -0.88 & $115.23 \pm 3.63$ & 2.786 & $109.23 \pm 3.63$ \\
\hline RG-BU 08-097 R & $5.120 *$ & $115.23 \pm 2.63$ & $5.120^{*}$ & $115.23 \pm 3.63$ & 3.12 & $108.90 \pm 3.63$ & $5.19 *$ & $113.23 \pm 3.63$ & -1.214 & $115.23 \pm 3.63$ \\
\hline RG-BU 08-105 R & 1.453 & $111.56 \pm 2.63$ & 1.453 & $111.56 \pm 3.63$ & 2.12 & $107.56 \pm 3.63$ & 3.12 & $112.23 \pm 3.63$ & -2.547 & $113.23 \pm 3.63$ \\
\hline Mean & & 107.54 & & 108.89 & & 111.87 & & 110.67 & & 111.58 \\
\hline$\overline{S E}(s i j)$ & & 4.126 & & & & & & & & \\
\hline SEd (Sij-Sik) & & 11.692 & & & & & & & & \\
\hline SEd (Sij-Skj) & & 13.205 & & & & & & & & \\
\hline $\mathrm{t}=S i j / S E I(S i j) a t$ & a) -2 & & & & & & & & & \\
\hline
\end{tabular}

$* \mathrm{p}=0.05, * * \mathrm{p}=0.01$ and $^{\mathrm{ns}}=$ non-significant 
Table 4. SCA effects (Sij) vis-à-vis per-se mean performance of hybrids for days to maturity in $80 \mathrm{~F}_{1}$ hybrids

\begin{tabular}{|c|c|c|c|c|c|c|c|c|c|c|}
\hline \multirow{3}{*}{$\begin{array}{l}\text { Line } \\
\text { Testers }\end{array}$} & \multicolumn{10}{|c|}{ Days to maturity } \\
\hline & \multicolumn{2}{|c|}{ IR 58025A } & \multicolumn{2}{|c|}{ GAN 46A } & \multicolumn{2}{|c|}{ IR 62829A } & \multicolumn{2}{|c|}{ IR 68888A } & \multicolumn{2}{|c|}{ BRRI 1A } \\
\hline & Sij effect & Mean & Sij effect & mean & Sij effect & mean & Sij effect & mean & Sij effect & Mean \\
\hline RG-BU 08-001 R & -3.92 & $139.64 \pm 6.87$ & $-5.29 *$ & $137.47 \pm 6.87$ & $-4.91 *$ & $140.85 \pm 6.87$ & $-4.43 *$ & $139.15 \pm 6.87$ & $-6.07 *$ & $137.55 \pm 6.87$ \\
\hline RG-BU 08-002 R & -1.25 & $142.31 \pm 6.87$ & 1.17 & $143.45 \pm 6.87$ & -0.11 & $144.73 \pm 6.87$ & -0.59 & $142.97 \pm 6.87$ & $6.64 *$ & $150.20 \pm 6.87$ \\
\hline RG-BU 08-005 R & $-6.40 *$ & $137.15 \pm 6.87$ & -1.13 & $142.97 \pm 6.87$ & -0.59 & $142.43 \pm 6.87$ & -1.59 & $141.97 \pm 6.87$ & $8.68 * *$ & $152.24 \pm 6.87$ \\
\hline RG-BU 08-006 R & $-9.23 * *$ & $134.33 \pm 6.87$ & $-8.23 * *$ & $144.97 \pm 6.87$ & 1.41 & $135.33 \pm 6.87$ & -1.88 & $141.68 \pm 6.87$ & $-13.26^{* *}$ & $130.30 \pm 6.87$ \\
\hline RG-BU 08-007 R & 1.70 & $145.26 \pm 6.87$ & 0.67 & $137.91 \pm 6.87$ & $-5.65^{*}$ & $144.23 \pm 6.87$ & -1.64 & $141.92 \pm 6.87$ & 3.21 & $146.77 \pm 6.87$ \\
\hline RG-BU 08-013 R & $-7.99 * *$ & $135.56 \pm 6.87$ & -2.02 & $139.90 \pm 6.87$ & -3.66 & $141.54 \pm 6.87$ & 2.84 & $146.40 \pm 6.87$ & 3.36 & $146.92 \pm 6.87$ \\
\hline RG-BU 08-016 R & $-9.35 * *$ & $134.20 \pm 6.87$ & 0.52 & $134.16 \pm 6.87$ & $-9.39 * *$ & $144.08 \pm 6.87$ & 1.68 & $145.24 \pm 6.87$ & $6.35^{*}$ & $149.91 \pm 6.87$ \\
\hline RG-BU 08-018 R & $-7.64 * *$ & $135.92 \pm 6.87$ & 3.69 & $135.13 \pm 6.87$ & $-8.43 * *$ & $147.25 \pm 6.87$ & -2.33 & $141.23 \pm 6.87$ & $8.37 * *$ & $151.93 \pm 6.87$ \\
\hline RG-BU 08-025 R & $-5.01 *$ & $138.54 \pm 6.87$ & $9.64 * *$ & $138.10 \pm 6.87$ & $-5.45^{*}$ & $153.20 \pm 6.87$ & 0.31 & $143.87 \pm 6.87$ & 3.03 & $146.60 \pm 6.87$ \\
\hline RG-BU 08-034 R & -3.76 & $139.80 \pm 6.87$ & 3.18 & $140.54 \pm 6.87$ & -3.02 & $146.74 \pm 6.87$ & 1.36 & $144.92 \pm 6.87$ & -1.46 & $142.10 \pm 6.87$ \\
\hline RG-BU 08-038 R & -1.01 & $142.55 \pm 6.87$ & 2.57 & $143.20 \pm 6.87$ & -0.36 & $146.13 \pm 6.87$ & 2.95 & $146.51 \pm 6.87$ & -2.25 & $141.30 \pm 6.87$ \\
\hline RG-BU 08-046 R & -0.56 & $143.00 \pm 6.87$ & $6.21 *$ & $142.99 \pm 6.87$ & -0.57 & $149.77 \pm 6.87$ & -0.23 & $143.33 \pm 6.87$ & 0.31 & $143.87 \pm 6.87$ \\
\hline RG-BU 08-057 R & 4.01 & $147.57 \pm 6.87$ & $8.26 * *$ & $146.60 \pm 6.87$ & 3.04 & $151.82 \pm 6.87$ & -1.33 & $142.23 \pm 6.87$ & -0.58 & $142.98 \pm 6.87$ \\
\hline RG-BU 08-063 R & 3.45 & $147.01 \pm 6.87$ & 2.91 & $145.37 \pm 6.87$ & 1.81 & $146.48 \pm 6.87$ & $5.27 *$ & $148.83 \pm 6.87$ & -1.24 & $142.32 \pm 6.87$ \\
\hline RG-BU 08-097 R & $-4.86^{*}$ & $138.42 \pm 6.87$ & -7.56 & $132.33 \pm 6.87$ & $-4.77 *$ & $140.07 \pm 6.87$ & $-7.21 * *$ & $136.77 \pm 6.87$ & $-5.34 *$ & $140.90 \pm 6.87$ \\
\hline RG-BU 08-105 R & 1.07 & $144.63 \pm 6.87$ & -2.59 & $145.30 \pm 6.87$ & 1.74 & $140.96 \pm 6.87$ & 2.32 & $145.88 \pm 6.87$ & 2.68 & $146.24 \pm 6.87$ \\
\hline Mean & & 140.99 & & 142.27 & & 145.35 & & 144.18 & & 145.01 \\
\hline$\overline{\boldsymbol{S E}}(s i j)$ & & 4.013 & & & & & & & & \\
\hline SEd (Sij-Sik) & & 6.870 & & & & & & & & \\
\hline $\begin{array}{l}\text { SEd (Sij-Skj) } \\
\mathrm{t}=\text { Sij/SEI (Sij) at }\end{array}$ & $d f=239$ & 7.442 & & & & & & & & \\
\hline
\end{tabular}

$* \mathrm{p}=0.05, * * \mathrm{p}=0.01$ and $^{\mathrm{ns}}=$ non-significant 
Table 5. SCA effects (Sij) vis-à-vis per-se mean performances of hybrids for grain yield (ton/ha) in $80 \mathrm{~F}_{1}$ hybrids

\begin{tabular}{|c|c|c|c|c|c|c|c|c|c|c|}
\hline \multirow{3}{*}{$\begin{array}{l}\text { Line } \\
\text { Testers }\end{array}$} & \multicolumn{10}{|c|}{ Grain yield } \\
\hline & \multicolumn{2}{|c|}{ IR 58025A } & \multicolumn{2}{|c|}{ GAN 46 A } & \multicolumn{2}{|c|}{ IR 62829A } & \multicolumn{2}{|c|}{ IR 68888A } & \multicolumn{2}{|c|}{ BRRI 1 A } \\
\hline & Sij effect & mean & Sij effect & mean & Sij effect & mean & Sij effect & mean & Sij effect & mean \\
\hline RG-BU 08-001 R & $0.88^{*}$ & $4.97 \pm 0.35$ & $0.57 *$ & $4.67 \pm 0.21$ & $1.16^{* *}$ & $5.26 \pm 0.22$ & $0.98^{* *}$ & $5.10 \pm 0.35$ & $1.97 * *$ & $6.06 \pm 0.35$ \\
\hline RG-BU 08-002 R & $2.67 *$ & $6.71 \pm 0.35$ & $3.50 * *$ & $7.59 \pm 0.35$ & $2.06^{* *}$ & $6.02 \pm 0.35$ & $1.24 *$ & $5.73 \pm 0.35$ & $1.23 * *$ & $5.86 \pm 0.35$ \\
\hline RG-BU 08-005 R & $-1.22 * *$ & $2.87 \pm 0.35$ & $-1.36^{* *}$ & $2.73 \pm 0.21$ & $2.35^{* *}$ & $6.44 \pm 0.35$ & $-2.91 * *$ & $1.18 \pm 0.21$ & $1.57 * *$ & $5.66 \pm 0.35$ \\
\hline RG-BU 08-006 R & $2.02 * *$ & $6.11 \pm 0.35$ & $1.17 * *$ & $5.91 \pm 0.21$ & $0.38^{*}$ & $4.48 \pm 0.21$ & $2.66 * *$ & $5.93 \pm 0.35$ & $1.47 * *$ & $5.62 \pm 0.35$ \\
\hline RG-BU 08-007 R & $1.55 * *$ & $5.53 \pm 0.35$ & $3.23 * *$ & $7.33 \pm 0.35$ & $1.54 *$ & $5.64 \pm 0.22$ & $1.62 * *$ & $5.71 \pm 0.35$ & $2.21 * *$ & $6.10 \pm 0.35$ \\
\hline RG-BU 08-013 R & $2.85 * *$ & $6.94 \pm 0.35$ & $-0.93 *$ & $3.16 \pm 0.21$ & $1.13 * *$ & $5.23 \pm 0.21$ & $-1.02 * *$ & $3.06 \pm 0.21$ & $-0.55^{*}$ & $3.53 \pm 0.21$ \\
\hline RG-BU 08-016 R & $-1.69 * *$ & $2.39 \pm 0.35$ & -0.35 & $3.74 \pm 0.21$ & $0.96 *$ & $5.05 \pm 0.21$ & $-0.70^{*}$ & $3.39 \pm 0.22$ & $3.39 * *$ & $7.48 \pm 0.35$ \\
\hline RG-BU 08-018 R & $1.26 * *$ & $5.36 \pm 0.35$ & $0.77 *$ & $4.86 \pm 0.21$ & $0.44 *$ & $4.53 \pm 0.21$ & $3.09 * *$ & $7.18 \pm 0.35$ & $-0.63 *$ & $3.46 \pm 0.21$ \\
\hline RG-BU 08-025 R & $-0.54 *$ & $3.54 \pm 0.35$ & 0.22 & $4.31 \pm 0.21$ & $2.23 * *$ & $6.33 \pm 0.35$ & $-2.18 * *$ & $1.91 \pm 0.21$ & $3.27 * *$ & $7.37 \pm 0.35$ \\
\hline RG-BU 08-034 R & 0.30 & $4.39 \pm 0.35$ & 0.31 & $4.40 \pm 0.21$ & $1.05 * *$ & $5.15 \pm 0.21$ & 0.33 & $4.43 \pm 0.22$ & $-0.91 *$ & $3.17 \pm 0.21$ \\
\hline RG-BU 08-038 R & $-0.71 *$ & $3.37 \pm 0.35$ & -0.08 & $4.01 \pm 0.21$ & $2.64 * *$ & $6.74 \pm 0.35$ & $-1.54 * *$ & $2.54 \pm 0.25$ & $-2.75 * *$ & $1.33 \pm 0.21$ \\
\hline RG-BU 08-046 R & $1.42 * *$ & $5.52 \pm 0.35$ & $-0.77 *$ & $3.32 \pm 0.21$ & $-0.47 *$ & $3.61 \pm 0.21$ & $-0.70 *$ & $3.38 \pm 0.21$ & -0.22 & $3.86 \pm 0.21$ \\
\hline RG-BU 08-057 R & $-2.81 * *$ & $1.27 \pm 0.35$ & $-2.26 * *$ & $1.83 \pm 0.21$ & $2.88 * *$ & $6.97 \pm 0.35$ & $0.84 *$ & $4.93 \pm 0.21$ & $0.89^{*}$ & $4.98 \pm 0.21$ \\
\hline RG-BU 08-063 R & $1.51 * *$ & $5.60 \pm 0.35$ & $1.54 * *$ & $5.63 \pm 0.21$ & $1.45 * *$ & $2.63 \pm 0.21$ & $0.81 *$ & $4.88 \pm 0.21$ & $1.57 * *$ & $5.66 \pm 0.21$ \\
\hline RG-BU 08-097 R & $2.95 * *$ & $6.73 \pm 0.35$ & $2.45 * *$ & $6.34 \pm 0.35$ & $1.83 * *$ & $6.26 \pm 0.35$ & $1.16^{* *}$ & $5.26 \pm 0.35$ & $2.21 * *$ & $6.18 \pm 0.35$ \\
\hline RG-BU 08-105 R & $1.77 * *$ & $5.86 \pm 0.35$ & 0.13 & $4.23 \pm 0.21$ & $-0.68 *$ & $3.41 \pm 0.22$ & -0.05 & $4.04 \pm 0.35$ & 0.01 & $4.10 \pm 0.21$ \\
\hline Mean & 4.08 & & 3.89 & & 4.67 & & 3.60 & & 4.20 & \\
\hline $\boldsymbol{S E}(s i j)$ & 0.354 & & & & & & & & & \\
\hline SEd (Sij-Sik) & 0.977 & & & & & & & & & \\
\hline SEd (Sij-Skj) & 1.104 & & & & & & & & & \\
\hline $\mathrm{t}=S i j / S E I(S i j) a t$ & $d f=239$ & & & & & & & & & \\
\hline
\end{tabular}

$* \mathrm{p}=0.05, * * \mathrm{p}=0.01$ and $^{\mathrm{ns}}=$ Insignificant 
As the above crosses showed positive SCA effects which could be used as above average combinations for tall stature. These results are in line with the findings of Roy \& Mandal (2001) and Singh \& Kumar (2004). Good specific combinations for tallness were evolved from high $\mathrm{x}$ high, general combiner parents. Low $\mathrm{x}$ above average general combiner parents produced above average specific combination for tall plant height in rest of the crosses. Xiao et al. (2003) also found similar findings.

Out of 80 test crosses fifteen crosses showed significant negative sca estimates for days to first flowering and twenty crosses showed significant negative sca estimates for days to maturity, where crosses of seven restorer with IR 58025A, two with GAN46A, six with IR 62829A, two with IR 68888A and three with BRRI 1A showed significant negative sca estimates for days to maturity. In all the cases it was observed that maximum number of crosses were found showing significant negative sca estimates with IR 58025A. The $\mathrm{F}_{1} \mathrm{~s}$ crosses of IR 58025A with seven restorer lines were observed significant negative sca estimates for days to first flowering, and seven $F_{1} s$ for days to maturity. As these combinations showed significant negative sca effects that could be used as above average specific combinations for earlier flowering. Hossain and Khoyumthem et al. 2005 in rice found significant negative sca values in days to $1^{\text {st }}$ flowering and maturity. From this table considering both sca effects and perse performances for days to $1^{\text {st }}$ flowering and maturity IR 58025A with RG-BU08-005R, RG-BU08-006R, RG-BU08-016R, RGBU08-018R and RG-BU08-025R might be recommended for earliness. These results are in line with the findings of Singh and Maurya 1999.

Ten crosses of IR 58025A with the restorers showed significant positive specific combining ability effects along with above average perse performances i.e., RG-BU 08-001R (0.881*, $4.97 \mathrm{t} / \mathrm{ha}), \mathrm{RG}-\mathrm{BU} 08-002 \mathrm{R}\left(2.67^{*}, 6.71 \mathrm{t} / \mathrm{ha}\right)$, RG-BU 08-006R (2.024**, 6.11 t/ha), RG-BU 08-013R (2.852**, 6.94 t/ha), RG-BU 08-018R (1.269**, 5.36 t/ha), RG-BU 08-046R (1.425**, 5.52 t/ha), RG-BU 08-063R (1.513**, 5.60 t/ha), RG-BU 08-063R (2.95**, 6.73 t/ha) and RG-BU 08-105R (1.774**, $5.86 \mathrm{t} / \mathrm{ha})$ for grain yield. Seven Crosses of GAN46A also showed highly significant positive sca effects and above average perse performances for grain yield were RG-BU 08-001R $\left(0.578^{*}, 4.67 \mathrm{t} / \mathrm{ha}\right)$, RG-BU 08-002R (3.50*, 7.59 t/ha), RG-BU 08-007R (3.426**, 7.33 t/ha), RG-BU 08-018R (0.722*, 4.86 t/ha), RGBU 08-063R (1.544**, 5.63 t/ha), RG-BU 08-097R (2.452**, 6.54 t/ha). Fourteen crosses of IR62829A resulted highly significant positive specific combining ability effects were found in the crosses with RG-BU08-001R (1.16**, 5.26 t/ha), RG-BU 08-002R (2.06*, 6.02 t/ha), RG-BU08-005R (2.350**, 6.44 t/ha), RG-BU08-013R (1.138**, 5.23 t/ha), RG-BU08-016R (0.960*, 5.05 t/ha), RG-BU08-025R (2.239**, 6.33 t/ha), RG-BU08-034R (1.056**, 5.15 t/ha), RGBU08-057R (2.885**, 6.97 t/ha), RG-BU08-063R (1.459**, $5.63 \mathrm{t} / \mathrm{ha})$ and RG-BU08-097R (1.83**, 6.26 t/ha). Nine crosses of IR68888A showed significant positive sca effects and above average perse performance were found in the crosses with RG-BU 08-001R (0.98**, 5.10 t/ha), RG-BU 08-006R (2.66*, 5.93 t/ha), RG-BU 08-007R (1.62**, 5.71 t/ha), RG-BU 08-018R (3.09**, 7.18 t/ha), RG-BU 08-057R (0.84**, 4.93 t/ha), RG-BU 08-063R (0.81**, 4.88 t/ha) and RG-BU 08-097R (1.16**, $5.26 \mathrm{t} / \mathrm{ha})$. Ten crosses of BRRI1A showed significant positive sca effects and above average perse performance were found in the crosses with RG-BU 08-001R (1.972**, 6.06 t/ha), RG-BU 08-002R (1.97*, 6.06 t/ha), RG-BU 08-005R (1.570**, $5.66 \mathrm{t} / \mathrm{ha}), \mathrm{RG}-\mathrm{BU}$ 08-006R (1.47**, 5.62 t/ha), RG-BU 08-007R (2.21**, 6.10 t/ha), RG-BU 08-016R (3.491**, 7.48 t/ha), RG-BU 08-025R (3.278**, 6.37 t/ha), RG-BU 08-063R (1.572**, $5.66 \mathrm{t} / \mathrm{ha})$ and RG-BU 08-097R (2.21**, $6.18 \mathrm{t} / \mathrm{ha})$. Khoyumthem et al. 2005 and Venkatesan et al. (2007) observed non-additive gene action governing the characters. Banumathy and Thiyagarajan 2005 also found similar results while studying sca variances of rice.

The crosses of five R-lines, RG-BU08-001R, RG-BU08-002R, RG-BU08-006R, RG-BU08-007R and RGBU08-097R were found resulting significant positive sca effects and above average perse performances with all five CMS lines for grain yield. RG-BU08-002R and RG-BU08-097R were found resulting significant positive sca effects with all yield contributing characters with all five CMS lines. Increased sca effect in yield might be due to significant positive sca values in pollen fertility, spikelets fertility, panicle exertion rate and significant negative sca values. Hossain and Khoyumthem 2005 found similar results in sca effects of several cross combinations. Chen et al. (2002) found high specific combinations of crosses of rice.

\section{REFERENCES}

Agrawal, K. B. 2003. Heterosis in rice. Annals of Agricultural Research. 24(2): 375-378.

Banumsathy, S. and K. Thiyagarajan. 2005. Heterosis of rice hybrids for yield and its yield components. Crop Res. Hisar. 25(2): 287-293.

Biju, S., S. Manonmani and K. Mohanasundaram. 2006. Studies on heterosis for yield and related characters in rice hybrids. Plant Archives. 6(2): 549-551. 
Chen, Y. J., X. H. Ding, G. Q. Zhang and Y. G. Lu. 2002. Studies on heterosis of $F_{1}$ hybrids in candidate Japonica lines in rice (Oryza sativa L.). J. of South China Agril. Univ. 23(4): 1-4.

Dorosti, H., A. J. Ali, G. Nematzadeh, H. Ghodsi and F. Alinia. 2006. IRRI, the first hybrid rice in Iran. International Rice Research Notes. 31(2): 31-32.

Faiz, F. A., M. Sabar, T. H. Awan, M. Ijaz and Z. Manzoor. 2006. Heterosis and combining ability analysis in Basmati rice hybrids. J. Animal and Pl. Sci. 16(1/2): 56-59.

Hossain, A., N. S. H. Mujtaba and F. J. Khoyumthem. 2005. The isolation and identification of volatile components from basmati rice (Oryza sativa L.). Flavor Science and Technology: Proc. $5^{\text {th }}$ Weurman Flavour Res. Symp. Wiley: New York.

Islam, M. A. 2009. Synchronization and stability analysis of hybrid seed production of rice in different environment. A Master Degree (MS) thesis of GPB Dept. BSMRAU, Salna, Gazipur.

Kempthorne, E. A. 1957. Biometrical Genetics, Combining ability through Line $\times$ Tester Method. Ed., 3. Chapman and Hall, London.

Khoyumthem, P., P. R. Sharma, N. B. Singh and M. R. K. Singh. 2005. Heterosis for grain yield and its component characters in rice (Oryza sativa L.). Environment and Ecology. 23 (Special 4): 687691.

Kumar, S., T. D. Senadhira and J. K. Chandrappa. 2007. Combining ability analysis for grain yield and other associated traits in rice. Oryza 44(2): 108-114.

Rao, A. M., S. Ramesh, R. S. Kulkarni, D. L. Savithramma and K. Madhusudhan. 2006. Heterosis and combining ability in rice. Crop Improvement, 23: 53-56.

Roy, A. K. and E. F. Mandal 2001. Development of aromatic cytosource for hybrid rice production. Ph. D. Dissertation, Bangabandhu Sheikh Muzibur Rahman Agricultural University, Gazipur 1706, Bangladesh. 166.

Salgotra, R. K., B. B. Gupta and P. Singh. 2009. Combining ability studies for yield and yield components in Basmati rice. Oryza 46(1): 12-16.

Shanthi, P., D. V. Yadav, A. K. Singh, G. Yadav and J. Singh. 2003. Genetic divergence for yield and its components in induced mutants of Mahsuri rice (Oryza sativa L.). Res. Corps. 2(3): 390-392.

Singh, R. J. and A. K. Kumar. 2004. Evaluation of CMS lines for various floral traits influence outcrossing in rice. International-Rice-Research-Notes; 28(1): 24-26.

Soni, D. K., K. Arvind and S. Lakeswar. 2005. Study of heterosis by utilizing cytoplasmic-genetic male sterility system in rice (Oryza sativa L.). Plant Archives. 5(2): 617-621.

Su, X. J. and C. H. Chen. 2006. Selection and utilization of a new rice restorer line Gui 1025 with small and high quality grains. Hybrid-Rice; 21(4): 21-23.

Tang, D. C., S. K. Huang, Y. G. Duan and Y. H. Wang. 2002. Studies on relationships of flowering time and pollination time with outcrossing rate of male sterile lines in hybrid rice seed production. Hybrid-Rice; 19(3): 50-54.

Venkatesan, N. D., D. M. Maurya, G. P. Verma and S. R. Vishwakarma. 2007. Heterosis for yield components in rice hybrids (Oryza sativa). I. J. Agril. Sci. 99(7): 1120-1122.

Virmani, S. S., J.B. Young, H. P. Moon, I. Kumar and J. C. Finn. 2000. Increasing Rice Yields through Exploitation of Heterosis. IRRI. Los Baños, Laguna, Philippines, p.12.

Xiao, G. Y., L. P. Yuan and L. Tang. 2003. Studies on heterosis of Indica/Javanica and Japonica/Javanica hybrids rice. Acta Agronomica Sinica. 29(2): 169-174.

Zhang, J., G. R. Chen, D. J. Huang, K. H. Liu and X. L. Tan. 2002. Genetic relationship of stigma exterior between maintainer lines and sterile lines for Dian type japonica hybrid rice. Journal-of-YunnanAgricultural-University; 20(4): 459-461, 477. 